\title{
LAS BOTIGUES DE TELES \\ DE BARCELONA: APORTACIÓN AL ESTUDIO DE LA OFERTA DE TEJIDOS Y DEL CRÉDITO AL CONSUMO (1650-1800) ${ }^{1}$
}

LIDIA TORRA FERNÁNDEZ

Universidad Pompeu Fabra

\section{RESUMEN}

El artículo describe la transformación de la venta al por menor a través de diversas botigues de teles de Barcelona con el fin de arrojar luz sobre la composición de la oferta textil, por una parte, y, por otra, la evolución del crédito comercial y al consumo. Se pone de relieve que a lo largo del periodo estudiado aumentó y se diversificó la oferta de tejidos y creció la capacidad del botiguer para destinar recursos a financiar la venta al fiado a sus clientes, entre los que se fue reforzando el protagonismo de los grupos menestrales.

\section{ABSTRACT}

This article describes the transformation of retail selling in different textile stores in Barcelona in order to throw light on the composition of the supply of textile goods and the evolution of commercial and consumer credit. The increase and diversification of the supply of textile goods are pointed out as well as the ability of the botiguers to finance sales on credit to their customers - with a growing presence of artisans and shopkeepers among them. JEL Classification: N33, N83.

${ }^{1}$ Esta investigación se llevó a cabo en el marco del proyecto DGICYT, PS 02-0120. Agradezco las sugerencias de los profesores Jaume Torras, Montserrat Durán y Enrique Llopis. Montserrat Durán me ha proporcionado información básica sobre terminología textil, y me ha facilitado la consulta del diccionario que prepara con Rosa Dávila Corona y Máximo García Fernández. Evidentemente, ellos no son responsables de los errores que pueda haber en el texto. 
El artículo presenta una descripción de las transformaciones acaecidas en la venta al por menor de tejidos en distintas botigues de teles de Barcelona, como vía de aproximación al análisis de la composición de la oferta textil, la evolución del crédito comercial y al consumo y los engranajes del sistema de distribución de bienes manufacturados en Cataluña entre 1650 y 1800. La documentación procedente del Archivo Histórico de Protocolos de Barcelona (AHPB) permite conocer detalladamente la actividad de las botigues de teles mediante protocolos de distinto carácter, como la formación de sociedades, los capítulos matrimoniales y testamentos, y sobre todo los inventarios. Éstos constituyen la base fundamental de este estudio, que se centra en la actividad de cuatro establecimientos distintos. Se trata, en primer lugar, de la botiga de Pau Rossell $(1668)^{2}$ y Joan Llinàs $(1686)^{3}$, socios ambos en una importante compañía comercial cuya actividad se prolongó durante varias décadas de la segunda mitad del siglo XVI ${ }^{4}$. En segundo lugar, Joan Pau Martí (1705) ${ }^{5}$ y su yerno Esteve Canals $(1756)^{6}$; en tercer lugar, Joan Galvany $(1737)^{7}$ y su yerno Joan Tarrida $(1758)^{8}$, y por último Lluís Aldever $(1758)^{9}$ y su hijo Pere Aldever (1792) ${ }^{10}$. Todos estos individuos regentaron establecimientos situados en la zona del Fossar de les Moreres y en las calles Canvis y Ample, en plena zona comercial de la ciudad.

El inventario de cada uno de ellos proporciona una imagen fija del negocio en un momento determinado. Su mayor virtud radica en la exhaustividad y el detalle con que describen cada pieza de tela de su almacén, consignando la denominación del nombre del tejido, el color o estampado, la longitud en canas ${ }^{11}$, la procedencia, y el precio por cana según tasación en el momento de realizar el inventario ${ }^{12}$. Su defecto, el silencio sobre aspectos para nosotros muy importantes, como cuánto tiempo llevaban las mercancías en el almacén, los ritmos de reposición, las ventas y balances anuales. La fuente ofrece, pues, una imagen exacta de las existencias pero

${ }^{2}$ Entre paréntesis figura el año en que se redactó el inventario.

AHPB, J. Sescases, concòrdies $i$ inventaris (1668-1679), fol. s/n.

${ }^{3}$ AHPB, G. Mora, concòrdies $i$ inventaris (1680-1692) (4), fol. s/n.

${ }^{4}$ Véase Lobato (1995), pp. 229-239.

5 AHPB, F. Rossell, manual d'inventaris $i$ encants (1702-1705), fol. 473.

${ }^{6}$ AHPB, A. Durán Quatrecases, llibre segon d'inventaris $i$ encants (1749-1758), fol. 245.

${ }^{7}$ AHPB, S. Pujol, llibre tercer d'inventaris $i$ encants (1737-1741), fol. 14.

${ }^{8}$ AHPB, S. Prats, llibre quart d'inventaris $i$ encants (1755-1758), fol. 371.

9 AHPB, S. Prats, llibre quart d'inventaris $i$ encants (1755-1758), fol. 483.

${ }_{10}$ AHPB, J. F. Avellá Navarro, protocolo (1792), fol. 314.

${ }^{11}$ Una cana son 8 palmos y equivale aproximadamente a 2 varas castellanas.

12 En todos los inventarios aparece junto al género la tasación monetaria del precio, excepto en el de Pere Aldever (1792). 
no del flujo de las mismas y sus tiempos de renovación. No obstante, cabe la posibilidad de comparar dos instantáneas de un mismo establecimiento, correspondientes a dos generaciones distintas. De ahí la oportunidad de profundizar, primero, en los cambios y permanencias de la composición del stock y formular, después, hipótesis relativas a la venta de tejidos a crédito, que se estudia en el segundo apartado de este trabajo.

\section{ESTRUCTURA Y COMPOSICIÓN DE LA OFERTA TEXTIL EN LAS BOTIGUES DE BARCELONA}

La tabla 1 pone de relieve los diversos órdenes de magnitud de las existencias de tejidos en los establecimientos analizados. Es importante subrayar el incremento del volumen de existencias que se da entre la pri-

\section{TABLA 1}

Volumen global de los tejidos en stock en cada establecimiento, clasificados según su procedencia (en canas y porcentajes)

\begin{tabular}{llrrrrr}
\hline & $\begin{array}{c}\text { Tejidos } \\
\text { importación } \\
\text { (canas) }\end{array}$ & $\%$ & $\begin{array}{c}\text { Tejidos } \\
\text { producción } \\
\text { autóctona } \\
\text { (canas) }\end{array}$ & $\%$ & $\begin{array}{c}\text { Volumen } \\
\text { global } \\
\text { (canas) }\end{array}$ \\
\cline { 3 - 7 } 1668 & Pau Rossell & 6.393 & 50,5 & 6.267 & 49,5 & 12.660 \\
1686 & Joan Llinàs & 9.444 & 49,0 & 9.830 & 51,0 & 19.274 \\
1705 & Joan Pau Martín & 19 & 2,0 & 926 & 98,0 & 945 \\
1756 & Esteve Canals & 2.269 & 33,0 & 4.605 & 67,0 & 6.874 \\
1737 & Joan Galvany & 3.547 & 46,0 & 4.163 & 54,0 & 7.710 \\
1758 & Joan Tarrida & 14.670 & 24,5 & 45.209 & 75,5 & 59.879 \\
1758 & Lluís Aldever & 744 & 26,0 & 2.117 & 74,0 & 2.861 \\
1792 & Pere Aldever & 2.924 & 12,0 & 21.444 & 88,0 & 24.368 \\
\hline
\end{tabular}

FUENTE:

AHPB, J. Sescases, concòrdies $i$ inventaris (1668-1679), fol. s/n.

AHPB, G. Mora, concòrdies i inventaris (1680-1692) (4), fol. s/n.

AHPB, F. Rossell, manual d'inventaris $i$ encants (1702-1705), fol. 473.

AIIPB, A. Duran Quatrecases, llibre segon d'inventaris $i$ encants (1749-1758), fol. 245.

AHPB, S. Pujol, llibre tercer d'inventaris $i$ encants (1737-1741), fol. 14.

AHPB, S. Prats, llibre quart d'inventaris $i$ encants (1755-1758), fol. 371.

AHPB, S. Prats, llibre quart d'inventaris $i$ encants (1755-1758), fol. 483.

AHPB, J. F. Avellá Navarro, protocolo (1792), fol. 314. 
TABLA 2

Distribución por fibras y origen de fabricación de los tejidos en stock en cada establecimiento (en porcentajes)

\begin{tabular}{|c|c|c|c|c|c|c|c|c|c|c|c|c|c|}
\hline & & \multicolumn{6}{|c|}{ Tejidos de importación } & \multicolumn{6}{|c|}{ Tejidos de producción autóctona } \\
\hline & & algodon & lana & lienzos & seda & mezcla & TOTAL & algodón & lana & lienzos & seda & mezcla & TOTAL \\
\hline 1668 & Pau Rossell & - & 28,5 & 21,0 & 1 & - & 50,5 & 1,5 & 36,0 & 5 & 6,5 & 0,5 & 49,5 \\
\hline 1686 & Joan Llinàs & 0,5 & 22,0 & 20,0 & 6 & 0,5 & 49,0 & 2,0 & 34,5 & 7 & 6,0 & 1,5 & 51,0 \\
\hline 1705 & Joan Pau Martí & - & 2,0 & - & - & - & 2,0 & - & 95,0 & - & 3,0 & - & 98,0 \\
\hline 1756 & Esteve Canals & 4,0 & 17,0 & 9,0 & - & 3,0 & 33,0 & 5,0 & 47,5 & 7 & 7,0 & 0,5 & 67,0 \\
\hline 1737 & Joan Galvany & 8,0 & 5,0 & 12,0 & 1 & 20,0 & 46,0 & 4,0 & 32,0 & 6 & 2,0 & 10,0 & 54,0 \\
\hline 1758 & Joan Tarrida & 5,0 & 4,0 & 10,0 & 1 & 4,5 & 24,5 & 7,5 & 24,0 & 17 & 6,0 & 21,0 & 75,5 \\
\hline 1758 & Lluís Aldever & 3,0 & 4,5 & 14,5 & - & 4,0 & 26,0 & 7,0 & 21,5 & 17 & 6,5 & 22,0 & 74,0 \\
\hline 1792 & Pere Aldever & 1,0 & 2,0 & 3,5 & 5 & 0,5 & 12,0 & 23,0 & 20,0 & 18 & 10,0 & 17,0 & 88,0 \\
\hline
\end{tabular}

FUENTE:

AHPB, J. Sescases, concòrdies $i$ inventaris (1668-1679), fol. s/n.

AHPB, G. Mora, concòrdies $i$ inventaris $(1680-1692)(4)$, fol. $\mathrm{s} / \mathrm{n}$.

AHPB, F. Rossell, manual d'inventaris $i$ encants (1702-1705), fol. 473.

AHPB, A. Duran Quatrecases, llibre segon d'inventaris i encants (1749-1758), fol. 245

AHPB, S. Pujol, llibre tercer d'inventaris $i$ encants (1737-1741), fol. 14.

AHPB, S. Prats, llibre quart d'inventaris $i$ encants (1755-1758), fol. 371

AHPB, S. Prats, llibre quart d'inventaris $i$ encants (1755-1758), fol. 483.

AHPB, J. F. Avellá Navarro, protocolo (1792), fol. 314 
mera y la segunda instantánea en todos y cada uno de los establecimientos estudiados. La misma tabla 1 muestra, en cada caso, además del volumen global de las existencias (en canas), el peso relativo de la oferta foránea y de la autóctona. Y en la tabla 2 se clasifican los tejidos de cada establecimiento según su fibra (o la mezcla de fibras). En líneas generales, la tabla 2 muestra las oscilaciones en la oferta de géneros de importación, que sin duda hay que relacionar con las medidas arancelarias ${ }^{13}$, pero también con un desplazamiento gradual de la oferta de tejidos de lana hacia géneros fabricados con fibras más ligeras y con texturas novedosas, como los tejidos de algodón, y un claro incremento de los tejidos de seda y géneros compuestos por la mezcla de lana o algodón con otras fibras.

En este sentido, los datos de que disponemos permiten pasar del análisis general al más particular. Empecemos por el establecimiento regentado por Rossell (1668) y Llinàs (1686). Prácticamente la mitad de las existencias contabilizadas en ambos inventarios eran de importación (tablas 1 y 2), procedían principalmente de los Países Bajos, Francia e Inglaterra (tabla 3), aunque lo significativo es el cambio de orientación de la oferta que se contempla en el período que transcurre entre las dos instantáneas. En efecto, parece ser que Rossell se especializó en la venta de paños de calidad media y paños finos procedentes de Flandes, mayoritariamente de Leiden, y también de núcleos industriales situados en Languedoc, concretamente de Carcasona y Lodève, así como también en la venta de sargas y camelotes producidos en Amiens y Sedan. Los lienzos provenían básicamente de Flandes y los paños de calidad, terciopelos y tejidos de seda llegaron al establecimiento barcelonés procedentes de distintas zonas europeas, comercializados a través de Génova y Marsella. Por su parte, los tejidos sencillos y más ordinarios llegaban básicamente de distintos núcleos industriales de Cataluña.

Entre las existencias del almacén de Joan Llinàs (1686), predominan distintas clases de lienzos y camelotes procedentes de los Países Bajos, algunas piezas de sargas, comercializadas a través de Londres, y los paños finos y de calidad media que llegaban de Carcasona. Lo más destacable es que, mayoritariamente, los paños y tejidos de menor calidad seguían siendo autóctonos. En este sentido, la documentación señala algunos centros productores catalanes como Berga, Camprodón, Ripoll y Sant Joan de les Abadesses; además de otros que fabricaban tejidos más finos como Sabadell, Barcelona o Reus. El documento sitúa en Barcelona la proce-

\footnotetext{
${ }^{13}$ Véase Fernández de Pinedo (1980), especialmente pp. 107-110 y 124-130.
} 


\section{TABLA 3}

Procedencia de los tejidos de importación en las botigues (en porcentajes)

\begin{tabular}{llcccc}
\hline & $\begin{array}{c}\text { Francia } \\
\%\end{array}$ & $\begin{array}{c}\text { Inglaterra } \\
\%\end{array}$ & $\begin{array}{c}\text { Países Bajos } \\
\%\end{array}$ & $\begin{array}{c}\text { Estados } \\
\text { Alemanes } \\
\%\end{array}$ & $\begin{array}{c}\text { Otros } \\
\%\end{array}$ \\
\cline { 2 - 6 } & 33 & 10 & 50 & - & 7 \\
1668 Pau Rossell & 3 & 6 & 88 & 2 & 1 \\
1686 Joan Llinàs & - & - & 100 & - & - \\
1705 Joan Pau Martí & 67 & 27 & 5 & - & 1 \\
1756 Esteve Canals & 48 & - & 22 & 19 & 11 \\
1737 Joan Galvany & 43 & 30 & 22 & 2 & 3 \\
1758 Joan Tarrida & 50 & 31 & 19 & - & -2 \\
1758 Lluís Aldever & 43 & 3 & 50 & 2 & 2 \\
1792 Pere Aldever & & & &
\end{tabular}

FUENTE:

AHPB, J. Sescases, concòrdies $i$ inventaris (1668-1679), fol. s/n. AHPB, G. Mora, concòrdies i inventaris (1680-1692) (4), fol. s/n. AHPB, F. Rossell, manual d'inventaris $i$ encants (1702-1705), fol. 473.

AHPB, A. Duran Quatrecases, llibre segon d'inventaris $i$ encants (1749-1758), fol. 245. AHPB, S. Pujol, llibre tercer d'inventaris $i$ encants (1737-1741), fol. 14. AHPB, S. Prats, llibre quart d'inventaris i encants (1755-1758), fol. 371. AHPB, S. Prats, llibre quart d'inventaris i encants (1755-1758), fol. 483. AHPB, J. F. Avellá Navarro, protocolo (1792), fol. 314.

dencia de los tejidos de lino, lino fino y algodón (tablas 2 y 4). En conjunto, los datos correspondientes a este establecimiento, tanto en la imagen de 1668 como en la de 1686 , muestran que cubría aquellos segmentos de la demanda donde la oferta local era deficiente, es decir, en los géneros de calidad media y alta. También es cierto que pone de manifiesto la revitalización de la manufactura autóctona durante estos años, con el progresivo aumento de la oferta de tejidos de lana, sobre todo sencillos y de calidad media ${ }^{14}$, elaborados a imitación de los foráneos.

El segundo establecimiento analizado corresponde a los primeros miembros de la familia Canals ${ }^{15}$. Aquí resulta visible el colapso que sufrió Bar-

\footnotetext{
${ }^{14}$ Véase Torres (1985), especialmente pp. 241-250, y Benaul (1992).

${ }_{15}$ Sobre la trayectoria de esta familia, véase Alier (1974), Thomson (1992), pp. 95 y ss., Vilar (1964-1968), vol. IV, pp. 431 y ss.
} 


\section{TABLA 4}

Número de tejidos distintos en las botigues (clasificados según la fibra y el origen de fabricación)

\begin{tabular}{|c|c|c|c|c|c|c|c|c|c|c|c|c|c|}
\hline & & \multicolumn{6}{|c|}{ Tejidos de importación } & \multicolumn{6}{|c|}{ Tejidos de producción autóctona } \\
\hline & & algodón & lana & lienzos & seda & mezcla & TOTAL & algodón & lana & lienzos & seda & mezcla & TOTAL \\
\hline 1668 & Pau Rossell & - & 17 & 8 & 3 & 一 & 28 & 1 & 7 & 8 & 5 & 1 & 22 \\
\hline 1686 & Joan Llinàs & 3 & 19 & 12 & 1 & 2 & 37 & 3 & 9 & 8 & 7 & 2 & 29 \\
\hline 1705 & Joan Pau Martí & 一 & 5 & 一 & 一 & - & 5 & - & 21 & - & 3 & 一 & 24 \\
\hline 1756 & Esteve Canals & 4 & 10 & 12 & 一 & 2 & 28 & 6 & 23 & 7 & 8 & 8 & 52 \\
\hline 1737 & Joan Galvany & 1 & 2 & 12 & 1 & 4 & 20 & 2 & 20 & 6 & 6 & 3 & 37 \\
\hline 1758 & Joan Tarrida & 3 & 3 & 14 & 3 & 6 & 29 & 5 & 23 & 9 & 9 & 8 & 54 \\
\hline 1758 & Lluís Aldever & 5 & 5 & 13 & - & 4 & 27 & 4 & 25 & 10 & 10 & 7 & 56 \\
\hline 1792 & Pere Aldever & 1 & 3 & 19 & 3 & 5 & 31 & 9 & 32 & 23 & 17 & 13 & 94 \\
\hline
\end{tabular}

FUENTE:

AHPB, J. Sescases, concòrdies $i$ inventaris (1668-1679), fol. $\mathrm{s} / \mathrm{n}$.

AHPB, G. Mora, concòrdies i inventaris (1680-1692) (4), fol. s/n.

AHPB, F. Rossell, manual d'inventaris $i$ encants (1702-1705), fol. 473.

AHPB, A. Duran Quatrecases, llibre segon d'inventaris $i$ encants (1749-1758), fol. 245.

AHPB, S. Pujol, llibre tercer d'inventaris $i$ encants (1737-1741), fol. 14.

AHPB, S. Prats, llibre quart d'inventaris $i$ encants (1755-1758), fol. 371.

AHPB, S. Prats, llibre quart d'inventaris $i$ encants (1755-1758), fol. 483.

AHPB, J. F. Avellá Navarro, protocolo (1792), fol. 314. 
celona en septiembre de 1705, tras el desembarco de las tropas aliadas. La botiga de Joan Pau Martí quedó devastada, y el inventario redactado en octubre de 1705 revela lo menguado de las existencias. Pero su contenido resulta interesante. En efecto, el almacén estaba surtido de bayetas y estameñas sencillas, y de géneros más bien bastos como barraganes, picotes y buratos, y también algunas piezas de paños de calidad entre baja y media, procedentes todos del país. En cambio, las pocas canas de tejido fino que se encontraba en el establecimiento eran de origen foráneo, concretamente, se trataba de paños y anascotes flamencos.

La situación cambió, y mucho, en los años que transcurrieron hasta el inventario de su yerno, Esteve Canals, realizado en 1756. En esta fecha, los tejidos de calidad media, finos y superfinos seguían llegando del extranjero. Se trataba de paños de distinta textura y calidad, y de anascotes producidos en Francia, Inglaterra y los Países Bajos ${ }^{16}$. Pero de forma paralela, se observa una presencia significativa de la manufactura autóctona con relación a la pañería de calidad media, y el incremento de la oferta en géneros de algodón y tejidos hechos con mezcla de fibras. Éstos, junto a las indianas, ocupan un lugar destacado en el almacén de Canals. Estos cambios ponen de manifiesto un activo espíritu comercial que se traducía en la oferta de artículos novedosos como las indianas, telería ligera, los tejidos de algodón y de mezcla de lino, seda o lana..., unos géneros que se adaptaban a los cambios de gustos y modas, algunas también importadas del extranjero. Todo ello muestra un entramado comercial bien organizado cuya característica principal radicaba en una oferta ampliamente variada (tabla 4).

La tercera botiga que se estudia en este trabajo es la que regentó primero Joan Galvany, fallecido en 1737, y luego Joan Tarrida, que murió en 1758. Quizás sea en este establecimiento donde mejor se observa la activa transformación de la manufactura catalana y su presencia cada vez más visible en las tiendas barcelonesas. Lo más destacable es que disminuyeron entre las dos fechas mencionadas los géneros de algodón y los tejidos de composición mixta de importación, a la vez que la oferta se mantenía en auge gracias a géneros de producción autóctona (tabla 2). En efecto, se estaba imitando con éxito la producción de diversas ramas manufactureras foráneas, que ampliaban la gama de géneros y variedades, como se puede observar en la tabla 4. Estas innovaciones tendrán una amplia aceptación

${ }^{16}$ Se observa una tendencia similar en distintos establecimientos situados en otras localidades catalanas; véase Torra (1998). 
en el mercado por las muchas ventajas que ofrecían las fibras y las nuevas texturas al consumidor, al ser transpirables, lavables, con mayor colorido y sobre todo con un precio menor. En este sentido, el desarrollo de nuevas técnicas de comercialización, junto al afán de los botiguers de teles por introducir géneros distintos y novedosos, exprimiendo los recursos de distribución y concesión de crédito, sentará las bases de un cambio en el comportamiento de los consumidores en el ámbito urbano que trascendió paulatinamente a las zonas rurales ${ }^{17}$.

Los inventarios que corresponden al último establecimiento analizado son una muestra de ello. Los géneros que se detallan en el inventario de Lluís Aldever, de 1758, son similares a los de otros establecimientos coetáneos, ya comentados. El gran cambio se produjo justamente en el período que le separa del inventario de su hijo Pere, redactado en 1792. Se observa un incremento considerable del volumen de existencias y, al mismo tiempo, un descenso drástico de los tejidos de importación fruto de unas medidas arancelarias efectivas y de la consiguiente sustitución de las importaciones por manufacturados autóctonos. El nuevo estado de cosas se refleja en los porcentajes relativos a la composición del stock de Pere Aldever (1792), en el que disminuye el peso relativo de los tejidos de lana de calidad media-alta de origen extranjero, mientras que aumenta la variedad de la oferta autóctona en tejidos de lana y géneros de mezcla, además de un incremento en lienzos y tejidos de algodón (tablas 1-4).

A modo de conclusión, señalamos algunos puntos que sobresalen de esta primera aproximación: incremento del stock, diversificación y cambio en la tendencia de la oferta textil en la trayectoria comercial de cuatro botigues de teles barcelonesas. El aumento generalizado del volumen global de tejidos que se da en cada caso entre la fecha del primer inventario y la del segundo indica, por un lado, el desarrollo de la demanda interior $y$, por otro, la capacidad de estos negocios para aumentar el tamaño de su oferta en el transcurso de pocos años. No sólo aumenta el volumen de tejidos, sino que el incremento mayor corresponde a los géneros de producción autóctona, y en este ámbito, a los géneros de algodón y tejidos compuestos por la mezcla de fibras distintas. La diversificación de la oferta es importante entre los géneros de lana, seda, telería, especialmente hacia mediados del Setecientos, y entre los tejidos de producción autóctona. Se percibe una preferencia creciente por los géneros con nuevas texturas, más ligeros y con mayor adaptabilidad a nuevos usos. En suma, se observa

\footnotetext{
${ }^{17}$ Algunos ejemplos en Torra (1997).
} 
un cambio gradual en la tendencia de la oferta textil en los establecimientos barceloneses que se acentúa hacia finales del siglo Xvin.

\section{EL CRÉDITO AL CONSUMO EN LAS BOTIGUES DE TELES}

La función crediticia del botiguer de teles contribuyó decisivamente a impulsar el aumento en la demanda. La venta al fiado era un instrumento importante para mantener una clientela fiel y estable, ampliar el radio comercial de su negocio, $y$, en definitiva, asegurarse futuras ventas. Para el consumidor, la compra a crédito fue un recurso fundamental para poder ampliar su consumo y para, hasta cierto punto, mantenerlo con un menor grado de dependencia de las oscilaciones del flujo de los ingresos.

En este sentido, los datos de los libros de cuentas de los inventarios permiten estudiar los cambios en la composición social de una parte importante de la clientela de estos comercios: los que en el momento de redactarse el documento figuraban como deudores del establecimiento. Nula es la información de la fuente acerca de los clientes que presumiblemente pagaban al contado, ni de los que pudieran haber saldado anteriormente su deuda. No obstante, incluso considerando estas limitaciones ${ }^{18}$, son centenares los nombres de individuos que recoge la documentación que junto con otros datos, como su procedencia, profesión y la suma del débito, permiten realizar un estudio aproximado de la tipología de la clientela, del radio comercial de cada establecimiento y del desarrollo del crédito al consumo en este sector. En este sentido, las tablas 5 y 6 agrupan los clientes deudores de cada establecimiento en once grupos socioprofesionales distintos con la finalidad de observar los colectivos que recurrieron en mayor escala al pago aplazado.

Los datos manejados ponen de manifiesto la existencia de una clientela estable y muy consolidada. Observamos familias enteras que, de generación en generación, acudían a hacer sus compras en el mismo establecimiento y pagaban los artículos a plazos: así, es corriente encontrar en estos documentos la referencia a individuos con débitos propios además de otras cantidades adeudadas por sus progenitores. Junto a esta clientela, llama la atención la presencia de nuevos actores sociales que progresivamente van engrosando las listas de clientes deudores (tabla 5). Se trata de diversos grupos menestrales urbanos, cuya presencia transformará gradualmente los patrones de la demanda.

\footnotetext{
${ }^{18}$ Véanse otros ejemplos en Rocha (1999).
} 
TABLA 5

Tipología de los clientes de las botigues de teles de Barcelona (según los débitos)

\begin{tabular}{|c|c|c|c|c|c|c|c|c|c|c|c|c|c|c|c|c|}
\hline & \multicolumn{2}{|c|}{$\begin{array}{l}\text { P. Rossell } \\
(1668)\end{array}$} & \multicolumn{2}{|c|}{$\begin{array}{l}\text { J. Llinàs } \\
(1686)\end{array}$} & \multicolumn{2}{|c|}{$\begin{array}{l}\text { J. P. Martí } \\
(1705)\end{array}$} & \multicolumn{2}{|c|}{$\begin{array}{l}\text { E. Canals } \\
(1756)\end{array}$} & \multicolumn{2}{|c|}{$\begin{array}{l}\text { J. Galvany } \\
(1737)\end{array}$} & \multicolumn{2}{|c|}{$\begin{array}{l}\text { J. Tarrida } \\
(1758)\end{array}$} & \multicolumn{2}{|c|}{$\begin{array}{l}\text { L. Aldever } \\
(1758)\end{array}$} & \multicolumn{2}{|c|}{$\begin{array}{l}\text { P. Aldever } \\
(1792)\end{array}$} \\
\hline & núm. & $\%$ & núm. & $\%$ & núm. & $\%$ & núm. & $\%$ & núm. & $\%$ & núm. & $\%$ & núm. & $\%$ & núm. & $\%$ \\
\hline Arrieros . & 2 & 0,5 & 3 & 2,0 & 4 & 1 & - & - & 2 & 4 & - & - & - & - & - & - \\
\hline Botiguers de teles............. & $4 \overline{3}$ & 12,0 & 45 & 14,0 & 19 & 7 & 29 & 16,0 & 11 & 20 & 59 & 14,0 & 36 & 20 & 77 & 15 \\
\hline Campesinos........................ & 3 & 0,5 & 11 & 3,0 & 13 & 5 & 4 & 2,0 & 4 & 8 & 2 & 0,5 & - & - & 一 & - \\
\hline Comerciantes-Nego- & 35 & 10.0 & 40 & 12.0 & 48 & 16 & 31 & 17,0 & 8 & 15 & 40 & 8,0 & 22 & 12 & 39 & 8 \\
\hline Marineros & 1 & 0,5 & - & - & - & - & - & - & - & - & - & - & - & - & - & - \\
\hline Menestrales ...................... & 89 & 25,0 & 86 & 27,0 & 93 & 32 & 64 & 35,0 & 16 & 31 & 156 & 33,0 & 36 & 21 & 186 & 35 \\
\hline Miembros órdenes re- & & & & & & & & & & & & & & & & \\
\hline 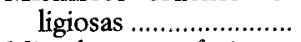 & 9 & 2,5 & 10 & 3,0 & 17 & 6 & 6 & 3,0 & - & - & 2 & 0,5 & 一 & - & 8 & 1 \\
\hline Miembros profesiones & & & & & & & & & & & & & & & & \\
\hline liberales........................ & 24 & 7,0 & 39 & 12,0 & 22 & 8 & 12 & 7,0 & 4 & 8 & 18 & 4,0 & 7 & 5 & 48 & 8 \\
\hline Militares ........................... & - & - & 1 & 0,5 & 2 & 1 & 1 & 0,5 & - & 一 & - & - & 11 & 6 & 143 & 27 \\
\hline Nobles............................. & 6 & 2,0 & 5 & 1,0 & 2 & 1 & 1 & 0,5 & 1 & 2 & 4 & 1,0 & 13 & 7 & 10 & 1 \\
\hline Servicio doméstico ......... & - & - & 1 & 0,5 & 3 & 1 & - & - & - & - & 一 & 一 & 一 & 一 & - & - \\
\hline $\begin{array}{l}\text { Total clientes que se co- } \\
\text { noce su oficio................. }\end{array}$ & 212 & 60,0 & 241 & 75,0 & 223 & 78 & 148 & 81,0 & 46 & 88 & 281 & 61,0 & 125 & 71 & 511 & 95 \\
\hline $\begin{array}{l}\text { Total clientes que se des- } \\
\text { conoce su oficio........ }\end{array}$ & 140 & 40,0 & 79 & 25,0 & 65 & 22 & 34 & 19,0 & 6 & 12 & 178 & 39,0 & 52 & 29 & 21 & 5 \\
\hline Total clientes deudores. & 352 & 100,0 & 320 & 100,0 & 288 & 100 & 182 & 100,0 & 52 & 100 & 459 & 100,0 & 177 & 100 & 532 & 100 \\
\hline
\end{tabular}

FUENTE:

AHPB, J. Sescases, concòrdies $i$ inventaris (1668-1679), fol. s/n.

AHPB, G. Mora, concòrdies $i$ inventaris $(1680-1692)(4)$, fol. $\mathrm{s} / \mathrm{n}$.

AHPB, F. Rossell, manual d'inventaris $i$ encants (1702-1705), fol. 473.

AHPB, A. Duran Quatrecases, llibre segon d'inventaris $i$ encants (1749-1758), fol. 245.

AHPB, S. Pujol, llibre tercer d'inventaris $i$ encants (1737-1741), fol. 14.

AHPB, S. Prats, llibre quart d'inventaris $i$ encants (1755-1758), fol. 371.

AHPB, S. Prats, llibre quart d'inventaris $i$ encants (1755-1758), fol. 483.

AHPB, J. F. Avellá Navarro, protocolo (1792), fol. 314. 
TABLA 6

Valor (media y mediana) del débito por cliente según su profesión en cada establecimiento (en lliures catalanes)

\begin{tabular}{|c|c|c|c|c|c|c|c|c|c|c|c|c|c|c|c|c|}
\hline & \multicolumn{2}{|c|}{$\begin{array}{c}\text { P. Rossell } \\
(1668)\end{array}$} & \multicolumn{2}{|c|}{$\begin{array}{c}\text { J. Llinds } \\
(1686)\end{array}$} & \multicolumn{2}{|c|}{$\begin{array}{l}\text { J. P. Martí } \\
(1705)\end{array}$} & \multicolumn{2}{|c|}{$\begin{array}{c}\text { E. Canals } \\
(1756)\end{array}$} & \multicolumn{2}{|c|}{$\begin{array}{c}\text { J. Galvany } \\
(1737)\end{array}$} & \multicolumn{2}{|c|}{$\begin{array}{c}\text { J. Tarrida } \\
(1758)\end{array}$} & \multicolumn{2}{|c|}{$\begin{array}{c}\text { L. Aldever } \\
(1758)\end{array}$} & \multicolumn{2}{|c|}{$\begin{array}{c}\text { P. Aldever } \\
(1792)\end{array}$} \\
\hline & media & mediana & media & mediana & media & mediana & media & mediana & media & mediana & media & mediana & media & mediana & media & mediana \\
\hline Arrieros & 53 & 43 & 6 & 6 & 11 & 8 & - & - & - & - & - & - & - & - & - & - \\
\hline Campesinos......................... & 9 & 4 & 8 & 7 & 6 & 3 & 7 & 7 & - & - & 6 & 6 & 7 & 6 & - & - \\
\hline $\begin{array}{l}\text { Botiguers de teles ............. } \\
\text { Comerciantes-Negocian- }\end{array}$ & 95 & 52 & 167 & 61 & 33 & 19 & 141 & 54 & 116 & 44 & 509 & 150 & 156 & 51 & 182 & 65 \\
\hline $\begin{array}{r}\text { tes ................................... } \\
\text { Miembros órdenes re- }\end{array}$ & 29 & 16 & 52 & 24 & 11 & 6 & 22 & 11 & 147 & 124 & 1.429 & 301 & 92 & 66 & 236 & 82 \\
\hline $\begin{array}{l}\text { ligiosas ......................... } \\
\text { Miembros profesiones }\end{array}$ & 6 & 5 & 41 & 9 & 25 & 4 & 13 & 16 & - & - & 10 & 8 & - & - & 58 & 11 \\
\hline 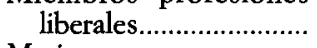 & 19 & 10 & 18 & 10 & 7 & 7 & 20 & 17 & 27 & 7 & 22 & 11 & 34 & 32 & 33 & 14 \\
\hline Marineros............................ & 2 & 2 & - & - & - & - & - & - & 一 & 一 & - & - & - & - & - & - \\
\hline Menestrales ...................... & 2 & 2 & 3 & 2 & 4 & 4 & 10 & 8 & 8 & 5 & 11 & 10 & 13 & 13 & 26 & 16 \\
\hline Militares ............................ & - & - & 11 & 11 & 5 & 4 & 4 & 4 & - & - & 31 & 10 & 41 & 11 & 40 & 16 \\
\hline (....................... & 60 & 33 & 111 & 38 & - & - & 144 & 101 & 81 & 61 & 471 & 320 & 36 & 21 & 181 & 56 \\
\hline Servicio doméstico ........... & 10 & 11 & 12 & 10 & 一 & - & 一 & - & - & - & 12 & 2 & - & - & 11 & 6 \\
\hline
\end{tabular}

FUENTE:

AHPB, J. Sescases, concòdies $i$ inventaris (1668-1679), fol. s/n.

AHPB, G. Mora, concòrdies i inventaris (1680-1692) (4), fol. $\mathrm{s} / \mathrm{n}$.

AHPB, F. Rossell, manual d'inventaris $i$ encants (1702-1705), fol. 473.

AHPB, A. Duran Quatrecases, llibre segon d'inventaris $i$ encants (1749-1758), fol. 245.

AHPB, S. Pujol, llibre tercer d'inventaris $i$ encants (1737-1741), fol. 14.

AHPB, S. Prats, llibre quart d'inventaris $i$ encants (1755-1758), fol. 371

AHPB, S. Prats, llibre quart d'inventaris $i$ encants (1755-1758), fol. 483.

AHPB, J. F. Avellá Navarto, protocolo (1792), fol. 314 . 
Otro aspecto que llama la atención en la tabla 5 es la presencia entre los deudores de otros botiguers de teles, así como de comerciantes, arrieros y negociantes que compraban para revender. El alto nivel de débito medio que se atribuye a estos sectores (tabla 6) muestra que los establecimientos estudiados contaban con una importante clientela redistribuidora de géneros textiles en la misma Barcelona, en otras poblaciones de Cataluña e incluso fuera del Principado (tabla 7). En efecto, conocemos los nombres y la ubicación geográfica de los botiguers de teles y comerciantes que compraban en estos establecimientos y lo que adeudaban, pero, en cambio, desconocemos las características concretas de los géneros que adquirían. Para ello, sería necesario localizar en la documentación notarial sus balances e inventarios y aun así sería muy difícil precisar exactamente los tejidos que les vendían los detallistas barceloneses. Una hipótesis plausible es que

\section{TABLA 7}

Distribución geográfica del volumen global del débito exclusivamente de otros botiguers de teles en cada establecimiento (en porcentajes)

\begin{tabular}{|c|c|c|c|c|c|c|c|}
\hline & & $\begin{array}{c}\text { Barcelona } \\
\text { ciudad } \\
\%\end{array}$ & $\begin{array}{c}\text { Barcelona } \\
\%\end{array}$ & $\begin{array}{c}\text { Girona } \\
\%\end{array}$ & $\begin{array}{c}\text { Tarragona } \\
\%\end{array}$ & $\begin{array}{l}\text { Lleida } \\
\%\end{array}$ & $\begin{array}{c}\text { Mercado } \\
\text { español } \\
\text { (excepto } \\
\text { Cataluña) } \\
\%\end{array}$ \\
\hline 1668 & Pau Rossell & 33 & 16 & 30 & 15 & 6 & - \\
\hline 1686 & Joan Llinàs & 44 & 19 & 24 & 9 & 4 & 一 \\
\hline 1705 & Joan Pau Martí & 96 & 3 & - & 1 & - & - \\
\hline 1756 & Esteve Canals & 27 & 39 & 13 & 8 & 9 & 4 \\
\hline 1737 & Joan Galvany & 58 & 23 & 7 & 2 & 5 & 5 \\
\hline 1758 & Joan Tarrida & 20 & 28 & 10 & 14 & 11 & 17 \\
\hline 1758 & Lluís Aldever & 29 & 38 & 15 & 12 & 2 & 4 \\
\hline 1792 & Pere Aldever & 10 & 50 & 5 & 11 & 4 & 20 \\
\hline
\end{tabular}

FUENTE:

AHPB, J. Sescases, concòrdies $i$ inventaris (1668-1679), fol. $\mathrm{s} / \mathrm{n}$.

AHPB, G. Mora, concòrdies $i$ inventaris $(1680-1692)(4)$, fol. $s / n$.

AHPB, F. Rossell, manual d'inventaris $i$ encants (1702-1705), fol. 473.

AHPB, A. Duran Quatrecases, llibre segon d'inventaris $i$ encants (1749-1758), fol. 245.

AHPB, S. Pujol, llibre tercer d'inventaris $i$ encants (1737-1741), fol. 14.

AHPB, S. Prats, llibre quart d'inventaris $i$ encants (1755-1758), fol. 371.

AHPB, S. Prats, llibre quart d'inventaris $i$ encants (1755-1758), fol. 483.

AHPB, J. F. Avellá Navarro, protocolo (1792), fol. 314. 


\section{TABLA 8}

Procedencia de los proveedores de las botigues de Barcelona (en porcentajes)

\begin{tabular}{|c|c|c|c|c|c|c|c|c|c|}
\hline & & $\begin{array}{c}\text { Barcelona } \\
\%\end{array}$ & $\begin{array}{c}\text { Resto } \\
\text { Cat. } \\
\%\end{array}$ & $\begin{array}{c}\text { Resto } \\
\text { Esp. } \\
\%\end{array}$ & $\begin{array}{l}\text { Francia } \\
\quad \%\end{array}$ & $\begin{array}{c}\text { Inglaterra } \\
\%\end{array}$ & $\begin{array}{c}\text { Génova } \\
\%\end{array}$ & $\begin{array}{c}\text { Ginebra } \\
\%\end{array}$ & $\begin{array}{c}\text { Otros } \\
\%\end{array}$ \\
\hline 1668 & Pau Rossell & 99 & 1 & - & - & - & 一 & - & - \\
\hline 1686 & Joan Llinàs & 99 & 1 & - & - & 一 & - & - & - \\
\hline 1705 & Joan Pau Martí & 100 & - & - & - & 一 & - & - & - \\
\hline 1756 & Esteve Canals * & - & - & - & - & 一 & - & - & - \\
\hline 1737 & Joan Galvany & 64 & - & - & 35 & 1 & - & - & - \\
\hline 1758 & Joan Tarrida & 28 & 5 & - & 65 & 2 & - & - & 一 \\
\hline 1758 & Lluís Aldever & 70 & 5 & - & 18 & 1 & 4 & 1 & 1 \\
\hline 1792 & Pere Aldever & 67 & 8 & 1 & 27 & 5 & 5 & 5 & 7 \\
\hline & $\begin{array}{l}\text { Sin datos. } \\
\text { UENTE: } \\
\text { HPB, J. Sescases, con } \\
\text { HPB, G. Mora, conce } \\
\text { HPB, F. Rossell, man } \\
\text { HPB, A. Duran Qua } \\
\text { HPB, S. Pujol, llibre } \\
\text { HPB, S. Prats, llibre } \\
\text { HPB, S. Prats, llibre }\end{array}$ & $\begin{array}{l}\text { ncòrdies } i \text { in } \\
\text { ordies } i \text { inve } \\
\text { rual d'inven } \\
\text { trecases, lli } \\
\text { tercer d'inve } \\
\text { quart d'inve } \\
\text { quart d'inve }\end{array}$ & $\begin{array}{l}\text { ventar } \\
\text { entaris } \\
\text { taris } i \\
\text { bre seg } \\
\text { entaris } \\
\text { entaris } \\
\text { entaris }\end{array}$ & $\begin{array}{l}\text { (1668 } \\
1680-1 \\
\text { encants } \\
\text { on d'inv } \\
i \text { encant } \\
\text { iencants } \\
\text { encant }\end{array}$ & $\begin{array}{l}3-1679), f \\
1692)(4), \\
(1702-17 \\
\text { ventaris } i \\
t s(1737-1 \\
\text { ts }(1755-1 \\
\text { ts }(1755-1\end{array}$ & $\begin{array}{l}\text { ol. s/n. } \\
\text { fol. s/n. } \\
\text { 05), fol. } 47 \\
\text { encants (174 } \\
\text { 1741), fol. } 1 \\
758 \text { ), fol. } 3 \\
\text { 758), fol. } 4\end{array}$ & $\begin{array}{l}3 . \\
19-1758), \\
4 . \\
71 . \\
83 .\end{array}$ & ol. 245 & \\
\hline
\end{tabular}

además de comprar toda clase de géneros, tuvieran especial interés en los tejidos más novedosos que marcaban el ritmo de la moda en ámbitos urbanos y tenían gran aceptación en el mercado catalán. Todo ello tendría su impacto en la consiguiente multiplicación cuantitativa y cualitativa de la demanda en zonas rurales, y en áreas alejadas de la capital con menor relevancia demográfica y económica ${ }^{19}$. De hecho, este proceso llegó a consolidarse gracias al importante crecimiento del mercado interior que favoreció la implantación y consolidación de puntos de venta estable y el

\footnotetext{
19 Véanse distintos ejemplos en Torra (2000).
} 
desarrollo del crédito y otros métodos de venta al por menor, ya arraigados en otras zonas europeas ${ }^{20}$.

En este sentido, es evidente que los canales de abastecimiento de estos botiguers partían a su vez de otros establecimientos, y de arrieros y negociantes que comercializaban directamente con los centros productores autóctonos, así como de mediadores y corresponsales ubicados en los principales núcleos industriales y comerciales europeos (tabla 8) ${ }^{21}$. Unos y otros tejían una red mercantil que cubría el país, cuyo epicentro eran los comercios de Barcelona. El desarrollo de esta estructura comercial constituye otro indicador de la ampliación de las relaciones de mercado en esta etapa $^{22}$.

\section{CONCLUSIÓN}

Durante la segunda mitad del siglo XVII, la presencia de la oferta extranjera en las botigues cubría especialmente aquellos segmentos donde la oferta autóctona era deficiente, principalmente los tejidos de calidad media-alta. En las décadas iniciales del siglo xvII se produjo un incremento de la oferta extranjera, tanto en términos absolutos como relativos, y un cambio de orientación de la manufactura autóctona, primero en el sector lanero y más tarde en el algodonero. Los productores autóctonos imitaban los tejidos extranjeros de mayor aceptación, principalmente franceses y flamencos, especialmente en géneros de lana y tejidos de mezcla con distintas fibras. Estas innovaciones, de todos modos, no adquirieron relieve en las botigues barcelonesas de tejidos hasta las décadas centrales del siglo XviII. Se constata una creciente diversificación del stock de tejidos y el incremento de la oferta en géneros de algodón, seda y en diferentes clases de lienzos. La base del éxito de estos artículos fue las distintas características de las fibras, además de otros elementos como el color, la textura y la adaptabilidad de los tejidos a nuevos usos, así como el factor moda, cuya influencia se fue acrecentando. El proceso tiene una doble vertiente: el progresivo aumento y diversificación de la oferta textil, ciertamente, pero también la capacidad del botiguer para invertir más recursos en la venta al fiado.

${ }^{20}$ Véanse, por ejemplo, McKendrick (1983); Hoh Cheung, Mui (1989); Benson, Shaw (1992), especialmente cap. 1; De Vries (1993); Muldrew (1993), especialmente pp. 171-173; Shammas (1990 y 1994).

${ }^{21}$ Véanse otros ejemplos en Torras i Ribé (1990).

22 Sobre estas transformaciones, véanse Vilar (1964-1968), vol. IV, pp. 165 y ss.; Fontana (1988), pp. 76 y ss.; Muset (1997), pp. 49 y ss.; Lluch (1996), pp. 93-119. 
Ello sin duda guarda relación con el protagonismo de los grupos menestrales, tanto si observamos su mayor afluencia a los establecimientos, como el aumento del valor medio de sus débitos.

\section{BIBLIOGRAFÍA}

ALIER, R. (1974): «La fàbrica d'indianes de la familia Canals», en Recerques, núm. 4, pp. 59-91.

Benaul, J. M. (1992): «Los orígenes de la empresa textil lanera en Sabadell y Terrassa en el siglo xvin», Revista de Historia Industrial, núm. 1, pp. 39-61.

Benson, J., y SHAw, G. (1992): The Evolution of Retail Systems c. 1800-1914, Leicester, Leicester University Press.

DE VRIES, J. (1993): «Between purchasing power and the world of goods: understanding the household economy in early modern Europe», en J. BREWER y R. PorTer (eds.), Consumption and the World of Goods, Londres-Nueva York, pp. $85-132$.

FeRNÁNDEZ DE Pinedo, E. (1980): «Coyuntura y política económica», en M. TuÑóN DE LARA (dir.), Historia de España. Centralismo, ilustración y agonía del Antiguo Régimen, Barcelona, Labor, pp. 11-173.

FonTANA, J. (1955): «Sobre el comercio exterior de Barcelona en la segunda mitad del siglo xvI», en Estudios de Historia Moderna, núm. 5, pp. 197-220.

- (1988): La fi de l'Antic Rèim i la industrialització, 1787-1868, Barcelona.

Hoh-Cheung, L., y MuI, L. (1989): Shops and shopkeeping in Eighteentb-Century England, Kingston, Magill-Queen's University Press.

LluCH, E. (1996): La Catalunya vençuda del segle XVIII. Foscors $i$ clarors de la Il.lustració, Barcelona, Edicions 62.

Lовато, I. (1995): Compañias y negocios en la Cataluña preindustrial, Sevilla, Universidad de Sevilla.

MCKendRICK, N. (1983): The Birth of a Consumer Society: the Commercialisation of Eighteentb-Century England, Londres, Hutchinson.

Molas Ribalta, P. (1974): «La Companyia Feu-Feliu de la Penya (1676-1708), comerç de teixits i estructura social vers 1700», en Cuadernos de Historia Económica, XII, pp. 77-126.

MuldREw, C. (1993): «Interpreting the Market: the Ethics of Credit and Community Relations in Early Modern England», en Social History, vol. 18, núm. 2, pp. 163-183.

MUSET, A. (1997): Catalunya $i$ el mercat espanyol al segle XVII: Els traginers $i$ els negociants de Calafi Copons, Barcelona, Publicacions de l'Abadia de Montserrat.

RoсHА, M. M. (1999): «Entre nosaltres n'hi ha prou amb la paraula»: les xarxes de crèdit no formal des d'una perspectiva històrica», en Recerques, núm. 39, pp. 171-192.

Shammas, C. (1990): The Pre-industrial consumer in England and America, Oxford, Clarendon.

- (1994): «The decline of textile prices in England and British America prior to industrialization», en Economic History Review, XLVII, pp. 483-507. 
Thomson, J. (1994): Els orígens de la industrialització a Catalunya: el cotó a Barcelona 1728-1832, Barcelona, Edicions 62.

Torra, L. (1997): «Comercialización y consumo de tejidos en Cataluña (1650-1800)», en Revista de Historia Industrial, núm. 11, pp. 177-196.

- (1998): «Comercio, difusión y consumo de tejidos de importación en Cataluña durante el siglo XvIII», en Pedralbes. Revista d'Història Moderna, núm. 18, pp. 73-82.

- (2000): «Botigues de teixits, crèdit comercial i crèdit al consum. Xarxes comercials a la Catalunya del segle xvin», en Recerques, núm. 41, pp. 5-30.

TORRAS I RIBÉ, J. M. (1990): «Els corresponsals del comerç català amb Europa al començament del segle xvII», en Recerques, núm. 23, pp. 115-139.

TORRES, X. (1985): «Activitats econòmiques», en Historia de Barcelona. Barcelona dins la Catalunya Moderna (segles XVI-XVII), vol. IV, Barcelona, Enciclopedia Catalana, pp. 215-255.

VILAR, P. (1964-1968): Catalunya dins l'Espanya Moderna, vols. III y IV, Barcelona, Edicions 62. 